\title{
Potential of Prevention Strategies for the Modifiable Risk Factor Type 2 Diabetes on the Future Number of Dementia Patients in Germany- A Multi- State Projection Through 2040
}

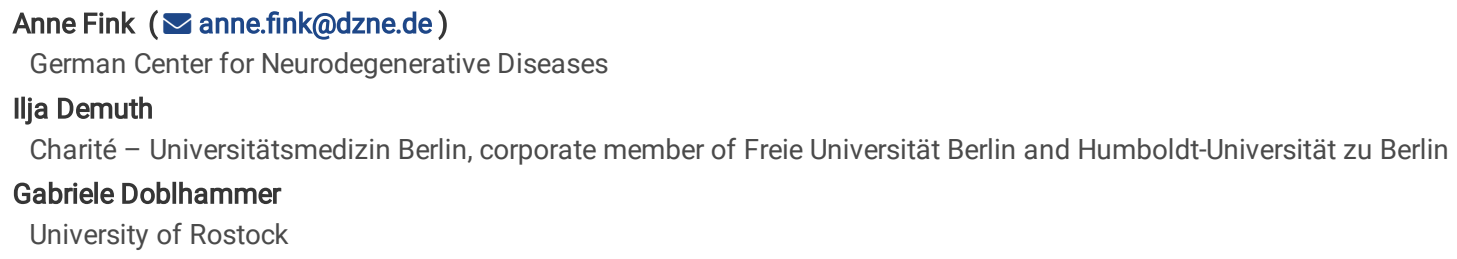

\section{Research Article}

Keywords: Diabetes, dementia, prevention strategies, forecast

Posted Date: June 2nd, 2021

DOI: https://doi.org/10.21203/rs.3.rs-538620/v1

License: @ (i) This work is licensed under a Creative Commons Attribution 4.0 International License. Read Full License 


\section{Abstract}

Background: We assess the impact of prevention strategies regarding type 2 diabetes as a modifiable risk factor for dementia and its consequences for the future number of dementia patients in Germany.

Methods: We used a random sample of health claims data $(\mathrm{N}=250,000)$ of insured persons aged $50+$ drawn in 2014 , and data on population size and death rates in 2015 from the Human Mortality Database. Using exponential hazard models, we calculated age- and sex-specific transition probabilities and death rates between the states (no diabetes/no dementia, diabetes/no dementia, no diabetes/dementia, diabetes/dementia). In multi-state projections, we estimated the future number of dementia cases aged 75+ through 2040 depending on the development of the incidence of diabetes among persons without diabetes and without dementia, and the dementia incidence among persons with and without diabetes.

Results: In 2015 there were 1.34 million people with dementia aged 75+ in Germany. A relative annual reduction in death rates of $2.5 \%$ will increase this number to 2.68 million by 2040 . A relative reduction of diabetes incidence by $1 \%$ annually would decrease dementia cases by around 26,000 , while a reduction of dementia incidence among people with diabetes by $1 \%$ would result in 175,000 fewer dementia cases. Both prevention strategies combined would prevent 191,000 dementia cases in 2040 .

Conclusions: The increase in life expectancy is decisive for the future number of people with dementia. Strategies of better diabetes treatment have the potential to lower the number of dementia patients in the coming decades.

\section{Background}

Dementia is one of the most important diseases of old age in terms of number of patients, resulting care need, and costs. To date there is no cure, and no curative treatment options are expected in the coming years [1,2]. A large body of literature, however, has shown the impact of modifiable risk factors on the incidence of dementia and their potential to prevent or at least to delay the onset of dementia and to postpone cognitive decline [3,4]. The management or avoidance of these risk factors can be summarized as primary and secondary prevention strategies [5].

Diabetes mellitus is one major modifiable risk factor of dementia. Numerous studies have linked type 2 diabetes with an increased risk of cognitive impairment and dementia [6-8]. About 3 to $4 \%$ of all Alzheimer's disease (AD) cases are attributable to diabetes [4], and diabetes patients have a 73\% increased risk of any dementia, a $56 \%$ increase of $A D$, and a $127 \%$ increase of vascular dementia ( $\mathrm{VaD})$ [7]. The mechanisms of how diabetes is associated with impaired cognitive function are not yet fully clarified. In addition to hyper- and hypoglycemic conditions, the main contributors are decreased insulin secretion, obesity, increased oxidative stress, and inflammation [9]. Diabetes induces vascular pathologies such as stroke, which in turn are risk factors of dementia, especially $\mathrm{VaD}$ [6]. In addition to its contribution to vascular diseases, there is some evidence that diabetes may directly cause AD [9]. Because dementia starts developing 15 to 20 years prior to clinical symptoms, midlife diabetes is of particular importance. Longitudinal studies found a significantly increased risk of dementia for persons with midlife diabetes $[10,11]$.

A considerable share of 12 to $14 \%$ of the adult population is affected by diabetes [12], and a substantial proportion lives with undiagnosed diabetes [13]. Studies estimated that diabetes prevalence increased in the past decades [12, 14], and projections for Germany indicate an additional increase [15].

A recent Cochrane review could not find definite proof for a special pharmacological therapy on the risk of cognitive decline and dementia [16]. However, some studies have indicated there is a protective effect of effective glycemic control via oral anti-diabetic medications on cognitive functioning [17, 18]. Furthermore, a review of diabetes-related dementia found promising evidence that regular physical activity and exercise might be beneficial for brain health in diabetes patients [19], and this is indicated by a number of studies (e.g. [20-22]). Espeland and colleagues found that a 24 months physical activity intervention was associated with improved performance of participants with diabetes in cognitive tests [23]. Additional evidence comes from animal experiments showing that exercise has positively impacted cognitive performance in a diabetes rat model [24]. However, additional research and especially randomized clinical trials in this field are undoubtedly warranted.

This study poses the question of whether preventing diabetes, or improving its treatment, has the potential to reduce the increasing number of dementia patients considerably through 2040. Preventing diabetes in mid-life may reduce the number of dementia patients in the long-term; better treatment of elderly diabetes patients may already have short to medium term effects by slowing down the progression rates to dementia. In order to answer this question, we try to quantify the effects of changes in the population structure (ageing of the baby boomers), increasing life expectancy, changes in diabetes incidence, and changes in the progression rates from diabetes to dementia, as well as from non-diabetes to dementia on the number of future dementia patients. We developed multi-state projections of the German population aged 75 and above through the year 2040, combining different assumptions about life expectancy, death rates and transition probabilities to depict possible effects of diabetes-prevention strategies, as well as improved diabetes-treatment options.

\section{Methods}

Human mortality database (HMD)

We used the Human mortality database (HMD, www.mortality.org), which provided information on the population size and death rates (50 to $89+$ years) in Germany in the year 2015.

Health claims data 
We used health claims data of the Allgemeine Ortskrankenkassen (AOK), the largest public health insurance company in Germany, to derive age- and sexspecific transition probabilities and mortality rate ratios. We used an age-stratified random sample of all AOK members in 2014 who had been born before 1965 (ages 50 years and above), which resulted in 250,000 insured persons; the sample was drawn by the data holder. Data were available until the end of 2017. The data consisted of demographic information on sex, date of birth, and date of death if applicable, as well as other information, and of medical information on all documented diagnoses and treatments of the inpatient and outpatient sectors. Diagnoses were coded by the German version of the 10 th revision of the International Statistical Classification of Diseases and Related Health Problems (ICD-10-GM). All information was available on a quarterly basis. After data cleaning, we thus had information on 249,966 insured persons including 111,624 men and 138,342 women. Comparison against the total German population by age and sex show a good representativeness of the study population. Only the proportions in the highest age groups are larger than in the overall German population (see Supplementary Figure S1, Additional file 1).

Definition of dementia

The following ICD-10 codes were used to define dementia: G30, G31.0, G31.82, G23.1, F00, F01, F02, F03, and F05.1. Because most dementia diagnoses are coded as "unspecified dementia" (F03), we do not differentiate by subtypes of dementia. Diagnoses in health claims data may be subject to legal changes or financial incentives. To overcome at least the problem of false-positive diagnoses [25], we applied a two-step validation strategy (see Supplementary section "Validation of diagnoses", Additional file 1). All subjects without a dementia diagnosis in 2014 and 2015 and a new diagnosis of dementia in 2016 or 2017 were assumed to be new cases.

Definition of type 2 diabetes

In the data set type 2 diabetes was defined as having one of the ICD-10 codes E11-E14 assigned. All subjects without a valid diabetes diagnosis in 2014 and 2015 and a new diagnosis of diabetes in 2016 or 2017 were assumed to be new cases.

Multi-state projection

To forecast the number of dementia patients aged 75 and above through the year 2040, we performed multi-state projections. We defined four transient states: 1. No diabetes / no dementia (Diab-Dem-), 2. Diabetes / no dementia (Diab+Dem)), 3. No diabetes / dementia (Diab-Dem $\left.{ }^{+}\right)$, 4. Diabetes / dementia $\left(\mathrm{Diab}^{+} \mathrm{Dem}^{+}\right.$), and 5. Death as an absorbing state (Figure 1). From the AOK data, we calculated transition probabilities by age group and sex between the states. Because both dementia and diabetes are chronic diseases, recurrent transitions rarely existed in the data and hence were not implemented in the multistate model.

Stata 16.0 was used for data management and regression analysis. We used PDE Population Module Software, developed by the Population Project of the International Institute for Applied System Analysis at Laxenburg (Austria) to perform our multi-state projections. We explored dementia cases from age 75 onwards, so we did not consider fertility rates and assumed a closed population without migration from 2015 onwards. Over the projection horizon of 25 years, the starting population (aged 50 years and above in 2015) was exposed to the sex-and age-specific transition probabilities and death rates resulting in a population aged 75 and above by 2040 (see Supplementary section "Calculation of population size in each state", Additional file 1).

Calculation of death rates $m_{x, t}$

To calculate the death rates $m_{x, t}$ we estimated age- and sex-specific mortality rate ratios of the four states based on AOK data. Therefore, we performed exponential hazard models for the risk of death for the whole study population and for the four states separately with age and the quadratic term of age as explaining variables.

Age- and sex-specific mortality rate ratios were calculated by dividing predicted age- and- sex-specific mortality rates of the four states by predicted age- andsex-specific mortality rates of the total study population. The resulting age- and- sex-specific mortality rate ratios were multiplied with HMD death rates to obtain state-specific death rates $m_{x, t}^{s}$ calibrated to the level of the total German population.

Calculation of the transition probabilities $t r$

First, age-specific hazard rates for the transitions (1) to (5) were derived separately by sex from exponential hazard models with age as well as the quadratic term of age as explaining variables. Then we used the predicted age-specific hazard rates $h_{x, t}^{s}$ and age-specific death rates $m_{x, t}^{s}$ to calculate separate agespecific transition probabilities ${ }^{t r_{x, t}^{s, s}}$ for men and women (see Supplementary section "Calculation of transition probabilities", Additional file 1).

Variants of assumptions

We modeled different scenarios with assumptions about the development of the transition probabilities and death rates. To explore the effect of changes in the age structure, we developed Scenario 1 (Sc1) with constant death rates and transition probability. We compared Sc1 with Scenario 2 (Sc2) to demonstrate the effect of increasing life expectancy. Here, we assumed a relative reduction of annual death rates in all states by $2.5 \%$. This assumption is comparable with modeled mortality reductions in the $14^{\text {th }}$ coordinated population projection of the German Federal Statistical Office [26].

Under the assumption of an increasing life expectancy as modeled in Sc2, we approximated strategies of primary and secondary prevention. Firstly, we incorporated changes only in one transition to explore the single effect of a prevention strategy on the number of future dementia cases (Scenarios 2.1 and 2.2). Subsequently, we combined prevention strategies (Scenarios 2.3). 
Scenario 2.1 (Sc2.1) 1 Diab-Dem $^{-} \rightarrow 2$ Diab $^{+}$Dem$^{-}$: To model the impact of a decreasing incidence of diabetes on the future number of dementia cases, we assumed a relative reduction of annual diabetes incidence of persons without dementia by $1 \%$. All other transitions did not change. In fact, data on temporal trends of diabetes incidence in Germany are limited [27]. However, since we would like to model prevention strategies a hypothetical annual reduction of $1 \%$ was assumed.

Scenario 2.2 (Sc2.2) $2 \mathrm{Diab}^{+} \mathrm{Dem}^{-} \rightarrow 4 \mathrm{Diab}^{+} \mathrm{Dem}^{+}$: Improvements in the treatment of diabetes regarding cognitive outcomes were accounted for by a relative reduction of annual age-specific dementia incidence of diabetes patients by $1 \%$ as well. Since the magnitude effect of a successful diabetes treatment in relation to dementia is not yet fully understood, we again assumed a hypothetical annual decrease in dementia incidence of $1 \%$ for persons with diabetes.

Scenario 2.3 (Sc2.3) 1 Diab-Dem ${ }^{-} \rightarrow 2$ Diab $^{+}$Dem $^{-}$and 2 Diab $^{+}$Dem $^{-} \rightarrow 4$ Diab $^{+}$Dem $^{+}$: We combined both prevention strategies regarding diabetes and simultaneously assumed an annual relative reduction in diabetes incidence of $1 \%$ in persons without diabetes and dementia and a relative reduction of dementia incidence of $1 \%$ per year in persons with diabetes.

Scenario 2.4 (Sc2.4) 1 Diab-Dem $^{-} \rightarrow 3$ Diab-Dem+: For comparison, we also modeled general unspecific dementia prevention strategies regarding the population without diabetes and dementia. Here, we assumed a relative reduction of age-specific dementia incidence by $1 \%$ annually. The Alzheimer Cohorts Consortium recently reported a $13 \%$ decline per decade in dementia incidence [28], which corresponds to a $1 \%$ reduction per year.

\section{Results}

In the base year 2015, there were 9 million people aged 75 years and above in Germany. At age $75,7.4 \%$ of men and $7.7 \%$ of women were affected by dementia. This share increased steeply with age, and in the highest age group aged 89 years and above $30.8 \%$ of men and $40.0 \%$ of women had a dementia diagnosis.

According to the prevalences from the AOK data (Table 1), 5.37 million people of this age group had neither a diagnosis of diabetes nor a diagnosis of

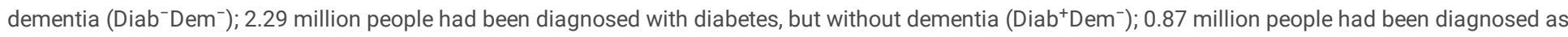
dementia patients without diabetes (Diab-Dem ${ }^{+}$); and 0.46 million people had a diagnosis of diabetes and dementia (Diab ${ }^{+}$Dem ${ }^{+}$). In total, 1.34 million people had received a dementia diagnosis (with and without diabetes diagnosis) in 2015.

Estimated transition probabilities

Figure 2 shows the estimated age- and sex-specific transition probabilities for the first year 2015 . At age 50 , about $1.2 \%$ of men and $0.8 \%$ of women of state 1 $\left(\right.$ Diab $\left.^{-} \mathrm{Dem}^{-}\right)$received a diabetes diagnosis within one year. Probabilities increase with age up to age 72 for men (2.2\%) and 76 for women (1.7\%); thereafter the incidence of diabetes decreases for both sexes. For the transition from state 1 (Diab-Dem ${ }^{-}$) to state 3 (Diab-Dem ${ }^{+}$) we observed an exponential increase for men and women, with slightly higher rates for men than for women. At age 50 , the probability to transit was $0.04 \%$ for men and $0.02 \%$ for women, at age $89+$ it was $5.6 \%$ for men and $5.3 \%$ for women. The probability to experience the transition to diabetes and dementia within one quarter was very low (state 1 to state 4). From age 80 onwards this probability was around $0.1 \%$ for men and women.

Regarding the transition from state $2\left(\mathrm{Diab}^{+} \mathrm{Dem}^{-}\right)$to state $4\left(\mathrm{Diab}^{+} \mathrm{Dem}^{+}\right)$, we observed an exponential increase for men and women, with slightly higher rates for men than for women until the age of 80 . At age 50 , the probability was $0.1 \%$, and in the highest age group of 89 years and older, probabilities were $9.2 \%$ for men and $10.3 \%$ for women. At all ages, the dementia probability of persons with diabetes was higher than for persons without diabetes. For the transition from state $3\left(\mathrm{Diab}^{-} \mathrm{Dem}^{+}\right)$to state $4\left(\mathrm{Diab}^{+} \mathrm{Dem}^{+}\right)$, we observed again a reverse U-shaped pattern with a probability of $4.4 \%$ for men at age 72 , and $4.0 \%$ for women at age 76.

\section{Estimated death rates}

Death rates (Fig. 3) rise exponentially with age, and individuals without diabetes and dementia (Diab-Dem-) had the lowest death rates at all ages, followed by those with diabetes only $\left(\mathrm{Diab}^{+} \mathrm{Dem}^{-}\right)$and dementia only $\left(\mathrm{Diab}^{-} \mathrm{Dem}^{+}\right)$. Patients with diabetes and dementia had the highest death rates. For example, at age 80 , the death rate of men of state $1\left(\mathrm{Diab}^{-} \mathrm{Dem}^{-}\right.$) was 0.04 , men of state $2\left(\mathrm{Diab}^{+} \mathrm{Dem}^{-}\right.$) had 0.05 , men of state 3 (Diab Dem $^{+}$) had $0.14 \mathrm{and}^{-1}$ the death rate of men with diabetes and dementia $\left(\mathrm{Diab}^{+} \mathrm{Dem}^{+}\right.$) was 0.16 . For women, corresponding values were $0.02,0.04,0.10$ and 0.12 (Fig. 3).

Results of multi-state projections

Modeling the demographic components of the future number of dementia patients reveals the overwhelming effect of future increases in life expectancy. Starting with 9 million individuals at ages 75 and above in 2015, the ageing of the baby boomer generation will lead to an increase of up to 10.89 million in 2040 , even under the assumption of constant transition probabilities and death rates (Sc1, Table 1). Rising life expectancy, modeled by a relative annual reduction of death rates in all states by $2.5 \%$, results in 13.45 million people (Sc2), which is comparable to the results of the 14 th coordinated population projection of the Federal Statistical Office in Germany [26]. 
Under the assumption of constant transition probabilities and death rates (Sc1) there will be a growth in the absolute number of persons with dementia, from 1.34 million in 2015 to 1.72 million in 2040 , corresponding to an increase of $29 \%$ compared to 2015 . Thus, the ageing of the baby boomers alone will add about 380,000 dementia patients. Relative annual reductions of death rates by $2.5 \%$ will additionally add almost one million dementia patients, totaling up to 2.68 million affected persons in 2040 (Sc2, Table 1 and Fig. 4).

In the following, we use the projections for 2040 from Sc2 as reference values.

In Sc2.1 (Table 1, Fig. 4), the 1\% relative annual reduction in diabetes incidence results in a reduced number of dementia cases with diabetes (Diab ${ }^{+}$Dem ${ }^{+}$: 1.50 million in Sc2.1-1.56 million in Sc2.2 = -60,000,-3.8\%), while dementia cases without diabetes (Diab-Dem $\left.{ }^{+}\right)$increase by $+3.0 \%(1.16$ million in Sc2.11.13 million in Sc2 $=+34,000$ ). This is because more people remain in state 1 under this assumption and thus are at risk of dementia. In sum, this strategy only slightly affects the future total number of dementia cases with 26,000 fewer cases (2.66 million in Sc2.1-2.68 million in Sc2=-26,000, $-1.0 \%$ ).

In Sc2.2, a relative reduction of $1 \%$ per year in the incidence of dementia in diabetes patients was assumed (Table 1, Fig. 4). This results in 1.38 million dementia cases with diabetes (Diab+Dem ${ }^{+}$: 1.38 million in Sc2.2-1.56 million in Sc2 $=-175,000,-11.3 \%$ ), while the trend in the number of dementia cases without diabetes $\left(\right.$ Diab $^{-} \mathrm{Dem}^{+}$) remains unchanged. Overall, this strategy results in 175,000 fewer dementia cases (2.51 million in Sc2.2-2.68 million in Sc2 = $-175,000,-6.5 \%)$.

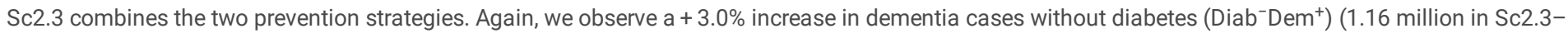
1.13 million in Sc2 $=+34,000$ ), but a 225,000 decrease in the number of individuals with both diagnoses to 1.33 million cases (Diab ${ }^{+}$Dem ${ }^{+}: 1.33$ million in Sc2.3-1.56 million in Sc2 = -225,000, -14.5\%). Combining the two prevention strategies yields 2.49 million dementia cases (2.49 million in Sc2.3-2.68 million in Sc2 $=-191,000,-7.1 \%)$.

In Sc2.4, a relative reduction of $1 \%$ per year in dementia incidence in persons without diabetes and dementia yields 0.96 million dementia cases without diabetes (Diab-Dem+: 0.96 million in Sc2.4-1.13 million in Sc2 = -170,000, -15.0\%) and 1.53 million dementia cases with diabetes (Diab ${ }^{+}$Dem ${ }^{+}$: 1.53 million in Sc2.4-1.56 million in Sc2 = -30,000, -1.5). Overall, general unspecific dementia prevention strategies result in 2.49 million dementia cases in 2040 (2.49 million in Sc2.4-2.68 million in Sc2 $=-193,000,-7.2 \%$ ). 
Table 1

Results of multi-state projection, estimated number of persons by state in Germany aged $75+$ in 2040

Projected number by state in million

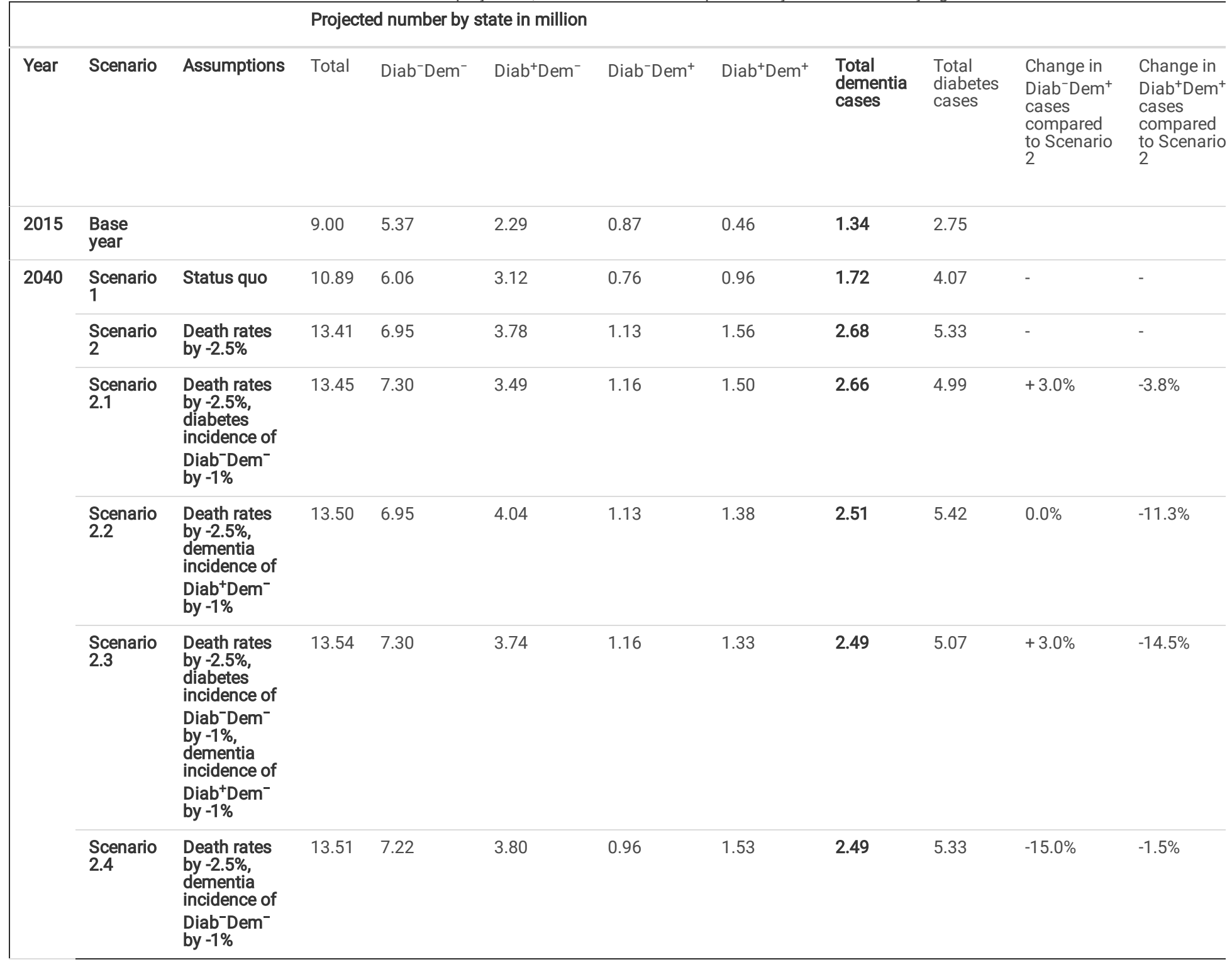

Note: Source: AOK 2014-2017 and HMD

\section{Discussion}

By 2040 , the future number of dementia patients aged 75 years and above will be mainly driven by the increase in life expectancy and changes in the age structure. Due to the changes in the age structure the number of dementia patients increases by one third (Sc1), while increasing life expectancy increases it by two thirds (Sc2).

When modeling the putative effect of interventions in the context of diabetes with a focus on dementia incidence in individuals without dementia and diabetes, two strategies can be pursued. First, the incidence of diabetes can be reduced, second the incidence of dementia can be reduced. The first strategy is depicted in Sc2.1. However, with 26,000 fewer dementia patients, the effect is marginal. This is the counterbalancing result of an increase in dementia patients without diabetes and a decrease in dementia patients with diabetes. Detrimental consequences of diabetes on cognition only accumulate over decades [10, $11]$, so that primary disease prevention measures only have their full protective effect in the long term and are not able to compensate for the baby boomer effect and the even much larger effect of increasing life expectancy in the medium term.

The second strategy is to reduce the incidence of dementia (Sc2.4), which might prevent about 193,000 dementia cases. This finding emphasizes the importance of also targeting risk factors other than diabetes in order to decrease the number of dementia patients.

Decreasing dementia incidence among diabetes patients (Sc2.2), would also have a sizeable and direct effect on the number of dementia patients. About 175,000 dementia patients could be prevented. Effective blood glucose control via diet, exercise and anti-diabetic medication may be key to these reductions [17-19, 29]. Combined implementation of both prevention strategies (Sc2.3) could prevent 191,000 dementia patients in 2040. 
Here, we only present the possible impact of one modifiable risk factor for dementia. In fact, the presence of diabetes is not independent from other potentially modifiable risk factors such as hypertension, physical inactivity, obesity, smoking, depression, or low educational attainment which are in turn also associated with dementia. Norton et al. showed that in Europe about 3.1\% of all AD cases were attributable to diabetes. For comparison, more than $20 \%$ of all AD cases were attributable to physical inactivity [4]. As we have no information on socio-economic and life style factors in our data, we cannot assess the impact of these factors on dementia.

Compared to other dementia projections for Germany, our results are at the upper boundaries. A recent study based on AOK data for Baden-Wurttemberg estimated there would be 1.8 to 2.9 million dementia cases for the total population in 2040 [30]. Baden-Wurttemberg is a federal state in Germany with one of the highest life expectancy rates and lowest morbidity. We used a random sample of all persons insured with the AOK in Germany, which may lead to higher dementia estimates. Another study based on meta-data of several population surveys projected there will be 2.4 to 2.6 million dementia cases aged $65+$ in 2040 [31]. However, this study assumed time-constant prevalence rates.

\section{Strengths and limitations}

The present study has several strengths and limitations. Our analyses rely on a high case number so that we can estimate transition probabilities and death rates up to the highest ages. Because of the routine documentation by physicians and hospitals, bias due to non-response, forgetfulness, interviewer bias, or self-selection can be ruled out. The institutionalized population is included regardless of their functional and cognitive status, which is particular important when analyzing dementia.

Health claims data are used primarily for billing purposes in health care and are not created for epidemiological analyzes. Therefore, documented diagnoses inevitably cannot reflect epidemiological disease development at the population level. First, persons who do not consult a doctor are not included in the diagnoses data. Second, coding errors and false-positive diagnoses are possible. To counteract this bias, we use an established two-stage validation procedure for dementia and diabetes.

While AOK claims data cover the total German population, the proportions of people with lower incomes and low education levels is higher than in other public health insurers in Germany, which leads to higher morbidity rates [32]. It is thus possible that these results may not be truly general for the total German population. However, we can assume that the correlations observed between the states are also true for the entire population.

\section{Implication for Public Health}

There will be a marked increase of persons with dementia within the next 20 years in Germany. We have shown the potential that the prevention and the better treatment of the modifiable risk factor type 2 diabetes might have to counterbalance some of this increase. There is a great deal of evidence that diabetes patients do not always adhere to treatment in terms of anti-diabetic medications or recommendations regarding diet and physical activity [33-36]. This holds true despite the fact that early detection of diabetes and subsequent treatment are essential, as the risk of dementia increases with the progression of diabetes [37]. Furthermore, only about half of the population without diabetes considers themselves to be well-informed about the causes and consequences of diabetes [38], which would be another important prerequisite for the primary prevention of diabetes with positive long-term consequences on the number of dementia patients.

From a societal point of view, reducing dementia is central because the illness is very care-intensive and therefore cost-intensive. There is a long period of care dependency over the course of the illness, as dementia patients spend $30 \%$ of their remaining life expectancy in the moderate stage of the illness, and $40 \%$ in the most severe stage of the illness [39]. Compared to previous cohorts, the baby boomer generation on average had fewer children who could provide care for parents. Thus, the future demographic development of the German population implies there will be an increasing imbalance between the number of potential caregivers and people with need for care [40].

\section{Conclusion}

Our results emphasize the need for prevention strategies of modifiable risk factors in order to lower the future number of dementia patients in Germany. We have focused on type 2 diabetes, which is only one major modifiable risk factor of dementia. If we succeed in reducing the incidence of additional risk factors such as hypertension, smoking, low education, or physical inactivity, then the reduction of the increase of the future number of dementia patients might be even higher. As most modifiable risk factors also contribute to other major diseases of the elderly, not only would dementia be prevented, but also cardiovascular disease and subsequent need for care and medical services. More research is needed to evaluate the effectiveness of prevention strategies and tailor these to the needs of the different strata of the population.

\section{Abbreviations}

$A D$

AOK

Diab-Dem

$\operatorname{Diab}^{+} \mathrm{Dem}^{-}$
Alzheimer's disease

Allgemeine Ortskrankenkassen

No diabetes / no dementia

Diabetes / no dementia 


$\begin{array}{ll}\text { Diab-Dem }^{+} & \text {No diabetes / dementia } \\ \text { Diab }^{+} \text {Dem }^{+} & \text {Diabetes / dementia } \\ \text { HMD } & \text { Human Mortality Database } \\ \text { ICD } & \text { International Statistical Classification of Diseases and Related Health Problem } \\ \text { Sc } & \text { Scenario } \\ \text { VaD } & \text { Vascular dementia }\end{array}$

\section{Declarations}

\section{Ethics approval and consent to participate}

All data of the Human Mortality Database (HMD) are publicly available on an aggregate basis. Consequently, no ethics approval was necessary.

Access to the health claims data was granted and approved by the Scientific Institute of the AOK (WIdO), while access to the complete database was not possible, due to current data protection regulations. All analyses of this study are based on anonymized administrative claims data which never identify patients directly. As individuals are anonymous, the results presented here do not in any way affect the persons whose records were used. No ethical approval was required, therefore this study complies with the tenets of the Declaration of Helsinki.

Consent for publication: Not applicable

Availability of data and materials

Human Mortality Database (HMD)

All data are publicly available from the Human Mortality Database (www.mortality.org).

\section{Health claims data}

The scientific research institute of the AOK (WIdO) has strict rules regarding data sharing because of the fact that health claims data are a sensitive data source and have ethical restrictions imposed due to concerns regarding privacy. Anonymized data are available to all interested researchers upon request. Interested individuals or an institution who wish to request access to the health claims data of the AOK may contact the WIdO (webpage: http://www.wido.de/, mail:wido@wido.bv.aok.de).

\section{Competing interests}

The authors declare that they have no competing interests.

\section{Funding}

None.

\section{Authors' contributions}

A.F. wrote the manuscript and researched data. G.D. wrote the manuscript and researched data. I.D. contributed to the introduction and discussion and reviewed/edited the manuscript. All authors read and approved the final manuscript.

\section{Acknowledgements}

We are grateful to the Scientific Research Institute of the AOK, WIdO, for providing the data. We would like to thank Renée Lüskow for English language editing.

\section{References}

1. Livingston G, Sommerlad A, Orgeta V, Costafreda SG, Huntley J, Ames D, et al. Dementia prevention, intervention, and care. Lancet. 2017;390(10113):2673-734.

2. Wimo A, Guerchet M, Ali G-C, Wu Y-T, Prina AM, Winblad B, et al. The worldwide costs of dementia 2015 and comparisons with 2010. Alzheimers Dement. 2017;13(1):1-7.

3. Fratiglioni L, Qiu C. Prevention of cognitive decline in ageing: dementia as the target, delayed onset as the goal. Lancet Neurol. 2011;10(9):778-9.

4. Norton S, Matthews FE, Barnes DE, Yaffe K, Brayne C. Potential for primary prevention of Alzheimer's disease: an analysis of population-based data. Lancet Neurol. 2014;13(8):788-94.

5. Katz D, Ali A. Preventive medicine, integrative medicine, and the health of the public. Commissioned IOM Summit Integr Med Health Public. 2009.

6. Biessels GJ, Staekenborg S, Brunner E, Brayne C, Scheltens P. Risk of dementia in diabetes mellitus: a systematic review. Lancet Neurol. 2006;5(1):64-74. 
7. Gudala K, Bansal D, Schifano F, Bhansali A. Diabetes mellitus and risk of dementia: a meta-analysis of prospective observational studies. J Diabetes Investig. 2013;4(6):640-50.

8. Cooper C, Sommerlad A, Lyketsos CG, Livingston G. Modifiable Predictors of Dementia in Mild Cognitive Impairment: A Systematic Review and MetaAnalysis. Am J Psychiatry. 2015;172(4):323-34.

9. Gaspar JM, Baptista FI, Macedo MP, Ambrósio AF. Inside the Diabetic Brain: Role of Different Players Involved in Cognitive Decline. ACS Chemical Neuroscience. 2016;7(2):131-42.

10. Beeri MS, Goldbourt U, Silverman JM, Noy S, Schmeidler J, Ravona-Springer R, et al. Diabetes mellitus in midlife and the risk of dementia three decades later. Neurology. 2004;63(10):1902-7.

11. Whitmer RA, Sidney S, Selby J, Johnston SC, Yaffe K. Midlife cardiovascular risk factors and risk of dementia in late life. Neurology. 2005;64(2):277-81.

12. Menke A, Casagrande S, Geiss L, Cowie CC. Prevalence of and trends in diabetes among adults in the united states, 1988-2012. J Am Med Assoc. 2015;314(10):1021-9.

13. Beagley J, Guariguata L, Weil C, Motala AA. Global estimates of undiagnosed diabetes in adults. Diabetes Res Clin Pract. 2014;103(2):150-60.

14. Danaei G, Finucane MM, Lu Y, Singh GM, Cowan MJ, Paciorek CJ, et al. National, regional, and global trends in fasting plasma glucose and diabetes prevalence since 1980: systematic analysis of health examination surveys and epidemiological studies with 370 country-years and $2 \cdot 7$ million participants. Lancet. 2011;378(9785):31-40.

15. Tönnies T, Röckl S, Hoyer A, Heidemann C, Baumert J, Du Y, et al. Projected number of people with diagnosed Type 2 diabetes in Germany in 2040. Diabetic Med. 2019;36(10):1217-25.

16. Sastre AA, Vernooij RW, Harmand MGC, Martínez G. Effect of the treatment of Type 2 diabetes mellitus on the development of cognitive impairment and dementia. Cochrane Database of Systematic Reviews. 2017(6).

17. Xu WL, von Strauss E, Qiu CX, Winblad B, Fratiglioni L. Uncontrolled diabetes increases the risk of Alzheimer's disease: a population-based cohort study. Diabetologia. 2009;52(6):1031-9.

18. Grodstein F, Chen J, Wilson RS, Manson JE. Type 2 diabetes and cognitive function in community-dwelling elderly women. Diabetes Care. 2001;24(6):1060-5.

19. Callisaya M, Nosaka K. Effects of exercise on type 2 diabetes mellitus-related cognitive impairment and dementia. J Alzheimers Dis. 2017;59(2):503-13.

20. Dybjer E, Nilsson PM, Engström G, Helmer C, Nägga K. Pre-diabetes and diabetes are independently associated with adverse cognitive test results: a crosssectional, population-based study. BMC Endocrine Disorders. 2018;18(1).

21. Fink A, Buchmann N, Tegeler C, Steinhagen-Thiessen E, Demuth I, Doblhammer G. Physical activity and cohabitation status moderate the link between diabetes mellitus and cognitive performance in a community-dwelling elderly population in Germany. PLoS One. 2017;12(10).

22. Buchmann N, Fink A, Tegeler C, Demuth I, Doblhammer G, Steinhagen-Thiessen E. Different treatment forms of type II diabetes and the risk of dementia in German health claims data. Acta Diabetologica. 2019;56(9):995-1003.

23. Espeland MA, Lipska K, Miller ME, Rushing J, Cohen RA, Verghese J, et al. Effects of physical activity intervention on physical and cognitive function in sedentary adults with and without diabetes. J Gerontol A Biol Sci Med Sci. 2017;72(6):861-6.

24. Mehta BK, Singh KK, Banerjee S. Effect of exercise on type 2 diabetes-associated cognitive impairment in rats. International Journal of Neuroscience. 2019;129(3):252-63.

25. Taylor J, Donald H, Østbye T, Langa KM, Weir D, Plassman BL. The accuracy of medicare claims as an epidemiological tool: the case of dementia revisited. J Alzheimers Dis. 2009;17(4):807-15.

26. Statisches Bundesamt. Bevölkerung Deutschlands bis 2060 - Ergebnisse der 14. koordinierten Bevölkerungsvorausberechnung - Hauptvarianten 1 bis 9 Wiesbaden: Statistisches Bundesamt; 2019.

27. Heidemann C, Scheidt-Nave C. Prevalence, incidence and mortality of diabetes mellitus in adults in Germany-a review in the framework of the diabetes Surveillance. Journal of Health Monitoring. 2017;2(3).

28. Wolters FJ, Chibnik LB, Waziry R, Anderson R, Berr C, Beiser A, et al. Twenty-seven-year time trends in dementia incidence in Europe and the United States: The Alzheimer Cohorts Consortium. Neurology. 2020;95(5):e519-e31.

29. Heneka MT, Fink A, Doblhammer G. Effect of pioglitazone medication on the incidence of dementia. Ann Neurol. 2015;78(2):284-94.

30. Milan V, Fetzer S. Die zukünftige Entwicklung von Demenzerkrankungen in Deutschland-ein Vergleich unterschiedlicher Prognosemodelle. Bundesgesundheitsblatt Gesundheitsforschung Gesundheitsschutz. 2019;62(8):993-1003.

31. Bickel H. Die Häufigkeit von Demenzerkrankungen. Informationsblatt Deutsche Alzheimer Gesellschaft e V Selbsthilfe Demenz [Internet]. 2016 05.03.2020:[1-5 pp.]. Available from: https://www.deutsche-alzheimer.de/unser-service/informationsblaetter-downloads.html.

32. Schnee M. Sozioökonomische Strukturen und Morbidität in den gesetzlichen Krankenkassen. In: Böcken J, Braun B, Amhof R, editors. Gesundheitsmonitor 2008: Gesundheitsversorgung und Gestaltungsoptionen aus der Perspektive der Bevölkerung: Verlag Bertelsmann Stiftung; 2008. p. 88-104.

33. Jarvie JL, Pandey A, Ayers CR, McGavock JM, Sénéchal M, Berry JD, et al. Aerobic Fitness and Adherence to Guideline-Recommended Minimum Physical Activity Among Ambulatory Patients With Type 2 Diabetes Mellitus. Diabetes Care. 2019;42(7):1333-9.

34. Polonsky WH, Henry RR. Poor medication adherence in type 2 diabetes: recognizing the scope of the problem and its key contributors. Patient Prefer Adherence. 2016;10:1299-307.

35. Jaworski M, Panczyk M, Cedro M, Kucharska A. Adherence to dietary recommendations in diabetes mellitus: disease acceptance as a potential mediator. Patient Prefer Adherence. 2018;12:163-74. 
36. Laxy M, Knoll G, Schunk M, Meisinger C, Huth C, Holle R. Quality of Diabetes Care in Germany Improved from 2000 to 2007 to 2014 , but Improvements Diminished since 2007. Evidence from the Population-Based KORA Studies. PLoS One. 2016;11(10).

37. Chiu W-C, Ho W-C, Liao D-L, Lin M-H, Chiu C-C, Su Y-P, et al. Progress of Diabetic Severity and risk of Dementia. J Clin Endocrinol Metab. 2015;100(8):2899-908.

38. Paprott R, Heidemann C, Stühmann LM, Baumert J, Du Y, Hansen S, et al. First results from the study 'Disease knowledge and information needs Diabetes mellitus (2017)'. Journal of Health Monitoring. 2018;3(S3):22-60.

39. Arrighi HM, Neumann PJ, Lieberburg IM, Townsend RJ. Lethality of Alzheimer disease and its impact on nursing home placement. Alzheimer Dis Assoc Disord. 2010;24(1):90-5.

40. Schulz E. Projection of care need and family resources in Germany. In: Doblhammer G, Scholz R, editors. Ageing, care need and quality of life. Wiesbaden: VS Verlag; 2010. p. 61 - 81.

\section{Figures}

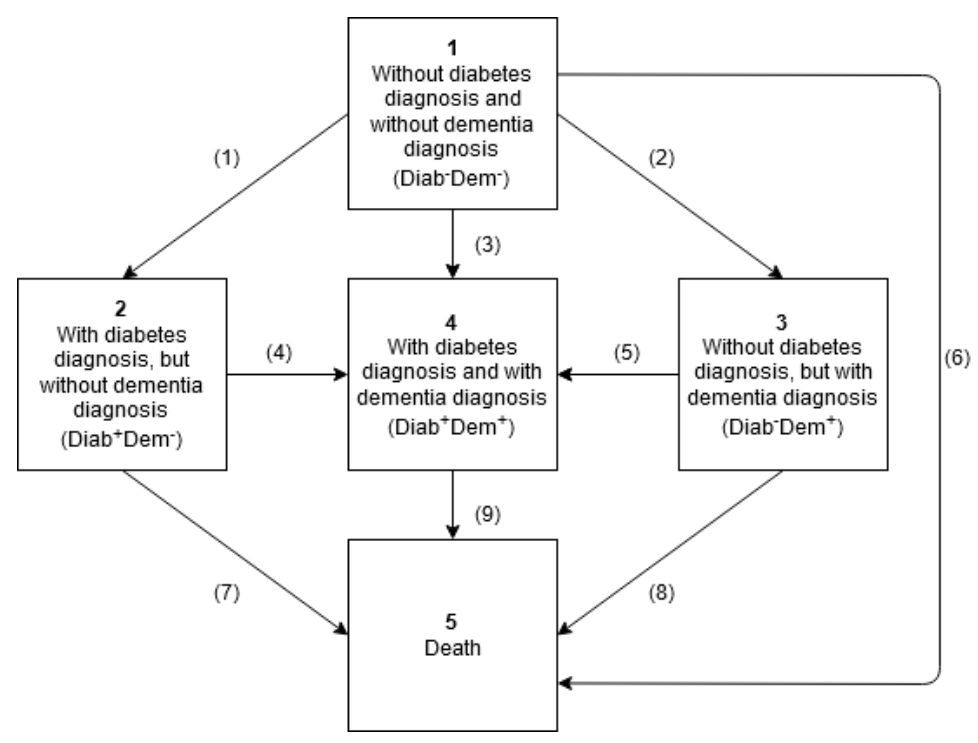

\section{Figure 1}

States and transitions for multi-state projection 

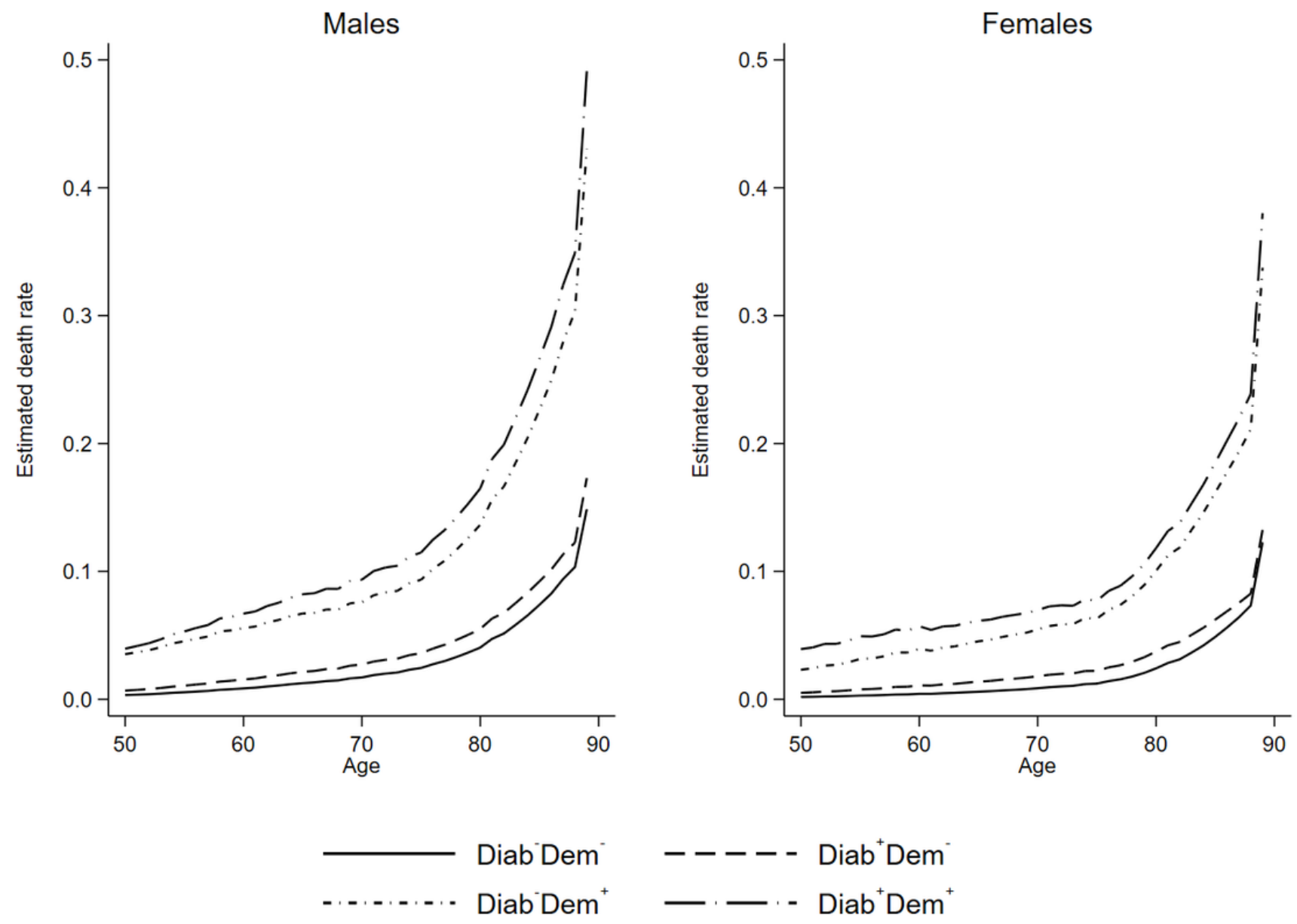

Figure 2

Estimated death rates from exponential hazard model by sex, age and state 

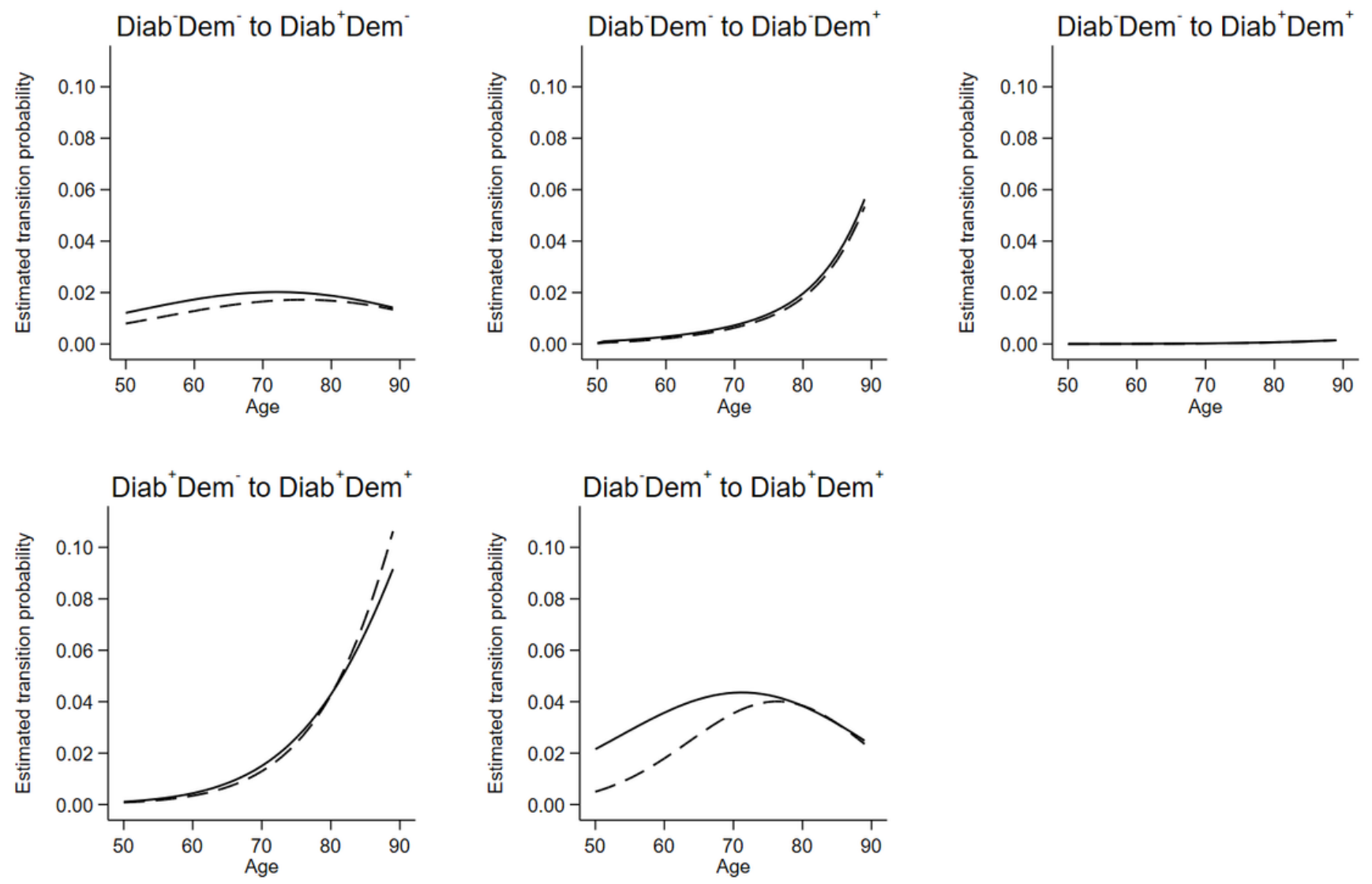

Males

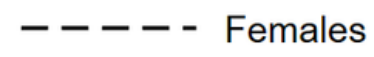

Figure 3

Estimated transition probabilities by sex and age 

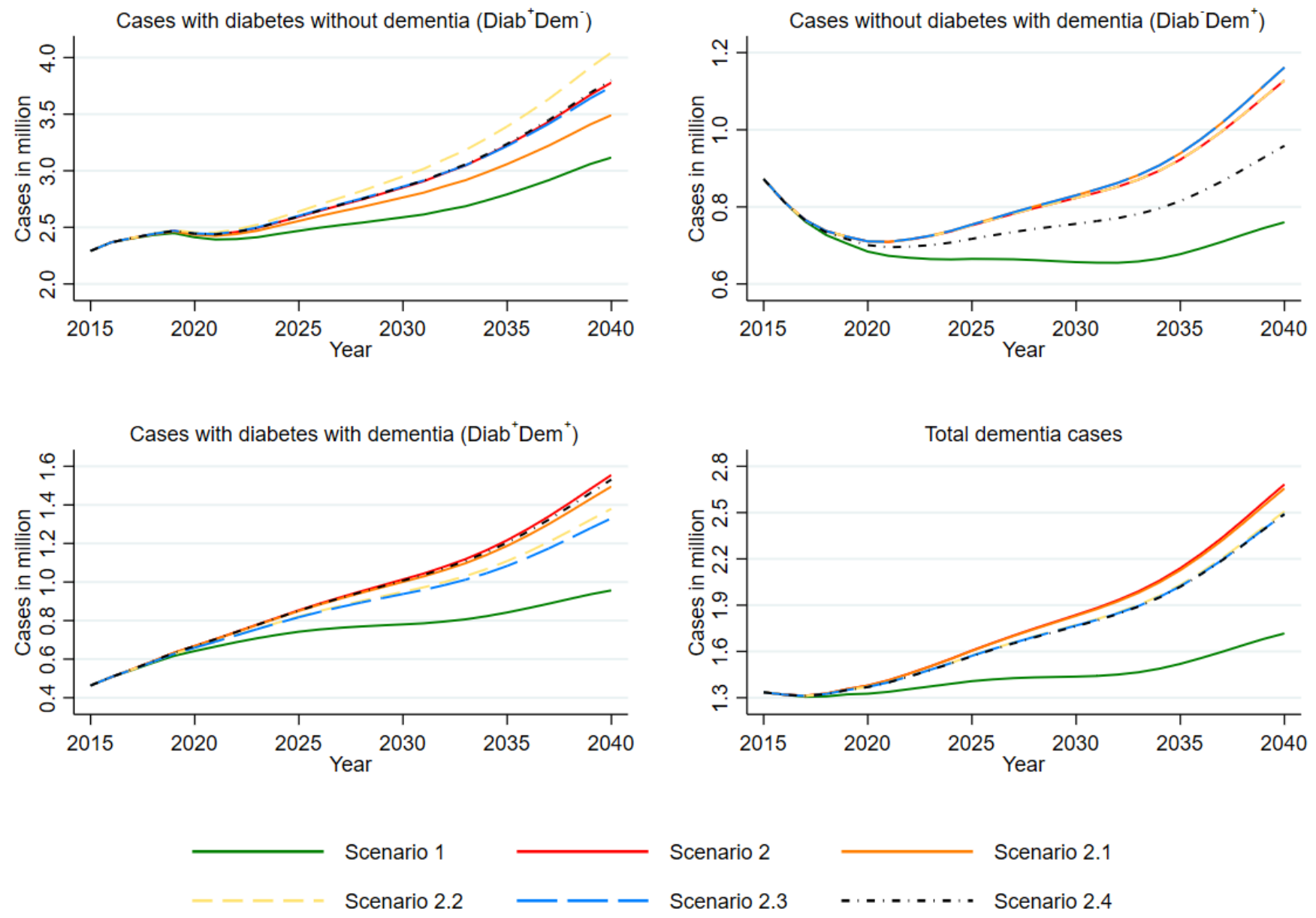

\section{Figure 4}

Results of multi-state projection, estimated number of cases by state and total dementia cases aged $75+$ through 2040

\section{Supplementary Files}

This is a list of supplementary files associated with this preprint. Click to download.

- Additionalfile1.docx 